\title{
THE COHESIVE LAW OF PARTICLE/BINDER INTERFACES IN SOLID PROPELLANTS
}

\author{
H. Tan \\ School of Engineering, University of Aberdeen \\ Fraser Noble Building, King's College, Aberdeen AB24 3UE, U.K.
}

Solid propellants are treated as composites with high volume fraction of particles embedded in the polymeric binder. A micromechanics model is developed to establish the link between the microscopic behavior of particle/binder interfaces and the macroscopic constitutive information. This model is then used to determine the tension/shearing coupled interface cohesive law of a redesigned solid rocket motor propellant, based on the experimental data of the stress-strain and dilatation-strain curves for the material under slow rate uniaxial tension.

\section{NOMENCLATURE}

$\begin{array}{ll}a & \text { particle radius } \\ E & \text { Young's modulus } \\ f & \text { particle volume fraction } \\ K & \text { bulk modulus } \\ P_{n} & \text { Legendre polynomial of mode } n \\ U & \text { strain energy density } \\ {[u]} & \text { radial displacement jump } \\ {[v]} & \text { tangential displacement jump } \\ \gamma_{\text {int }} & \text { total interface cohesive energy per unit area } \\ \Delta & \text { volume dilatation } \\ \delta_{\text {open }} & \text { critical opening displacement of the interface } \\ \delta_{\text {slide }} & \text { critical sliding displacement of the interface } \\ \bar{\varepsilon} & \text { macroscopic axial strain } \\ \theta & \text { angle from axial direction } \\ \lambda & \text { combined measure of interface displacement jump } \\ \lambda_{e} & \text { linear coefficient in interface cohesive energy } \\ \mu & \text { shear modulus }\end{array}$

This is an Open Access article distributed under the terms of the Creative Commons Attribution-Noncommercial License 3.0, which permits unrestricted use, distribution, and reproduction in any noncommercial medium, provided the original work is properly cited. 


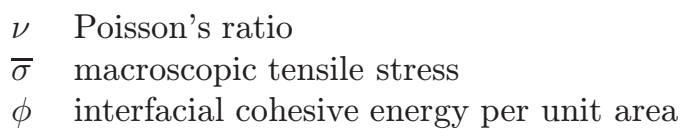

\section{INTRODUCTION}

Modern solid propellants consist of very high volume fraction of particles of different sizes in polymeric binder. The specific surface (i.e., high interface area per unit volume of solid propellant) is very high such that the behavior of particle/binder interfaces may significantly influence the macroscopic behavior of composite materials [1]. The interfacial debonding in a solid propellant governs the damage behavior such as fracture. It was observed that the macroscopic crack propagation is mainly along the particles/binder interface in solid propellants [24]. The debonding of the interfaces close to the burning surface can cause sudden transition from surface to volumetric burning, thus triggering deflagration-todetonation transition.

Behavior of particle/binder interfaces affects the sensitivity, and thus safety related issues, of solid propellants. Obtaining a constitutive law of the solid propellant that accounts for nonlinear interface debonding is crucial in wholesystem computational simulation of solid rockets to predict safety and reliability. While it is difficult to directly measure the particle/binder interface cohesion with loading applied to a plastic bonded energetic material, indirect method using macroscopic experimental data was developed [5]. This research aims at determining the interfaces cohesive law in a solid propellant based on macroscopic constitutive information obtained experimentally.

Experimental data for macroscopic constitutive behavior of solid propellants are available. Figure 1 shows the stress-strain and volume dilatation-strain relations for a redesigned space-shuttle solid rocket motor (RSRM) propellant subject to uniaxial tension at a low strain rate [6]. The RSRM propellant consists of ammonium perchlorate $(69.7 \%$ (wt.)), aluminum $(16 \%$ (wt.)), iron oxide $(0.3 \%$ (wt.)), poly(butadiene-acrylonitrile) (PBAN) polymer (12.04\%(wt.)), and epoxy curing agent (1.96\%(wt.)). Particle/binder interface debonding is the major damage mechanism that causes yielding in Fig. $1 a$ and volume dilatation increase in Fig. $1 b$.

The objective of this paper is to determine the interface cohesive law using the RSRM propellant constitutive data. In section 2, the tension-shearing coupled interface cohesive law is proposed with four parameters to be determined. In section 3, a micromechanics model, extended Mori-Tanaka method accounting for interface debonding, is used to link the microscopic interface jumps with the macroscopic stress-strain and dilatation-strain information during a uniaxial tensile test. Using the model, the values of the four interface parameters are obtained from the constitutive experimental data, as presented in section 4 . 


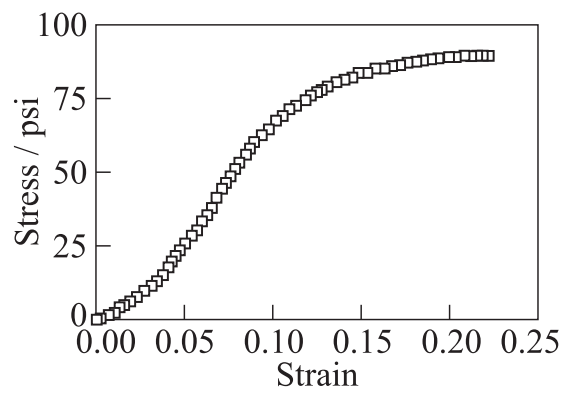

(a)

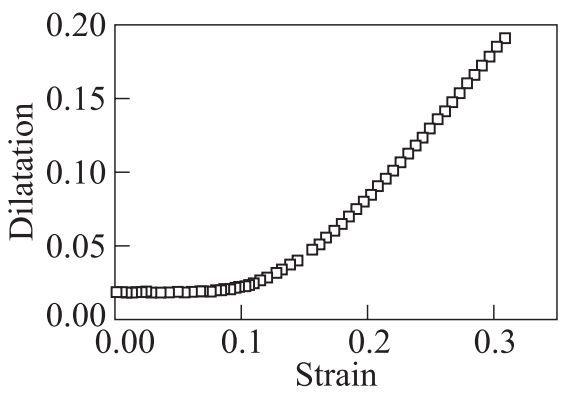

(b)

Figure 1 Stress-strain and dilatation-strain curves for an RSRM propellant subject to uniaxial tensile load at strain rate $0.0714 / \mathrm{min}$ [6]: (a) tensile stress vs. axial strain; and $(b)$ dilatation vs. axial strain

\section{INTERFACE COHESIVE MODEL}

At the particle/binder interface, there are radial displacement jumps $[u]$ and tangential displacement jump $[\nu]$. The interfacial cohesive energy per unit area, $\phi$, depends on a combined measure of interface displacement jump:

$$
\lambda=\sqrt{\left(\frac{[u]}{\delta_{\text {open }}}\right)^{2}+\left(\frac{[v]}{\delta_{\text {slide }}}\right)^{2}}
$$

where $\delta_{\text {open }}$ and $\delta_{\text {slide }}$ are the critical opening and sliding displacements of the interface, respectively, and $\lambda=1$ corresponds to complete interface debonding. For pure interface opening $([v]=0),[u]=\delta_{\text {slide }}$ is the start of complete debonding. For pure interface sliding, $([u]=0),[v]=\delta_{\text {slide }}$ is the start of complete debonding. The energy $\phi$ is assumed to depend on $\lambda$ only $[7], \phi=\phi(\lambda)$.

Similar to the expression of particle/binder interface cohesive energy as a function of $\lambda$ for a plastic bonded explosive [8], $\phi(\lambda)$ for RSRM propellant is assumed to take the form

$$
\phi(\lambda)= \begin{cases}\left(\frac{\lambda}{\lambda_{e}}\right)^{2} \gamma_{\mathrm{int}} & \text { if } \lambda<\lambda_{e} ; \\ {\left[1-\frac{(1-\lambda)^{2}}{1-\lambda_{e}}\right] \gamma_{\mathrm{int}}} & \text { if } \lambda_{e} \leq \lambda \leq 1 ; \\ \gamma_{\mathrm{int}} & \text { if } \lambda>1\end{cases}
$$

where $\gamma_{\text {int }}$ is the total interface cohesive energy per unit area, and $\lambda_{e}$ is the linear coefficient. 


\section{MICROMECHANICS MODEL \\ LINKING INTERFACE BEHAVIOR WITH CONSTITUTIVE INFORMATION}

Consider a representative volume element (RVE) of solid propellant material consisting of spherical particles of different radius $a_{I}$ (with volume fraction $f_{I}$ ), and subject to uniaxial tension of axial strain $\bar{\varepsilon}$. Each particle is embedded in a homogenized media subject to uniaxial tension, thus the distribution of interface displacement jump can be approximated as axisymmetric. Therefore, the opening displacement jump $\left[u^{I}\right]$ and tangential displacement jump $\left[v^{I}\right]$ can be expanded in Legendre polynomial series as

$$
\begin{aligned}
& {\left[u^{I}\right]=\sum_{n=0}^{\infty}\left[u_{n}^{I}\right] P_{n}(\cos \theta) ;} \\
& {\left[v^{I}\right]=\sum_{n=2}^{\infty}\left[v_{n}^{I}\right] \frac{d P_{n}(\cos \theta)}{d \theta}}
\end{aligned}
$$

where $\theta=0$ and $\theta=\pi$ are along the axial direction; $\left[u_{n}^{I}\right]$ and $\left[v_{n}^{I}\right]$ are the mode- $n$ Legendre polynomial coefficients; and the summation, here and thereafter, is for even numbers only.

The extended Mori-Tanaka method accounting for nonlinear interface debonding in a composite subject to fixed tensile strain $\bar{\varepsilon}$ gives the strain energy density of the RVE as [9]:

$$
U=\frac{\bar{\sigma}^{2}}{2 E_{0}}-\frac{3 e_{0} \varepsilon_{0}^{2}}{2 M_{0}^{u}}-\frac{3 e_{2} \varepsilon_{2}^{2}}{4\left(M_{2}^{u}+3 M_{2}^{v}\right)}+\frac{3}{2} \sum_{I} \frac{f_{I}}{a_{I}}\left(\phi_{I}^{\Omega}+\int_{0}^{\pi} \phi_{I} \sin \theta d \theta\right)
$$

where $\phi_{I}$ is the cohesive energy of interfaces around a particle of radius $a_{I}$, and $\phi_{I}^{\Omega}$ is defined as

$$
\phi_{I}^{\Omega}=\sum_{n=0}^{\infty} \frac{\left(M_{n}^{u}+M_{n}^{v}\right)\left[u_{n}^{I}\right]^{2}-2 n(n+1) M_{n}^{v}\left[u_{n}^{I}\right]\left[v_{n}^{I}\right]+n(n+1) M_{n}^{u}\left[v_{n}^{I}\right]^{2}}{(2 n+1)\left[M_{n}^{u}+(n+1) M_{n}^{v}\right]\left(M_{n}^{u}-n M_{n}^{v}\right) a_{I}}
$$

where $M_{n}^{u}$ and $M_{n}^{v}$ are the mode- $n$ material properties related to mode- $n$ Legendre polynomial coefficients [9], the mode- 0 and mode- 2 interfacial strains are defined as

$$
\begin{aligned}
& \varepsilon_{0}=\sum_{I} f_{I} \frac{\left[u_{0}^{I}\right]}{a_{I}} \\
& \varepsilon_{2}=\sum_{I} f_{I} \frac{2\left(\left[u_{2}^{I}\right]+3\left[v_{2}^{I}\right]\right)}{5 a_{I}}
\end{aligned}
$$


respectively. Mode- 0 and mode- 2 coefficients are defined as

$$
\begin{aligned}
e_{0} & =\frac{\bar{M}_{0}}{(1-f) M_{0}^{u \sigma}+f \bar{M}_{0}} ; \\
e_{2} & =\frac{\bar{M}_{2}}{(1-f)\left(M_{2}^{u \sigma}+3 M_{2}^{v \sigma}\right)+f \bar{M}_{2}}
\end{aligned}
$$

where $f=\sum_{I} f_{I}$ is the total volume fraction of particles, and

$$
\begin{aligned}
& \bar{M}_{0}=\frac{1}{3 K_{b}}+\frac{1}{4 \mu_{b}} \\
& \bar{M}_{2}=\frac{15\left(1-\nu_{b}\right)}{2\left(7-5 \nu_{b}\right) \mu_{b}}
\end{aligned}
$$

where $K_{b}, \mu_{b}$ and $\nu_{b}$ are the bulk modulus, shear modulus, and Poisson's ratio, respectively, of the binder during slow loading. The macroscopic tensile stress $\bar{\sigma}$ relates to the macroscopic axial strain $\bar{\varepsilon}$, mode- 0 and mode- 2 interfacial strains through

$$
\bar{\sigma}=E_{\text {perf }}\left(\bar{\varepsilon}-e_{0} \varepsilon_{0}-e_{2} \varepsilon_{2}\right) .
$$

Here, $E_{\text {perf }}$ is the Young's modulus for the same composite with perfect interfaces (without bonding):

$$
E_{\text {perf }}=\left(\frac{1}{E_{b}}+\frac{f}{3}\left\{\left(\frac{1}{3 K_{p}}-\frac{1}{3 K_{b}}\right) e_{0}+\left(\frac{1}{\mu_{p}}-\frac{1}{\mu_{b}}\right) e_{2}\right\}\right)^{-1}
$$

where $E_{b}$ is the Young's modulus of the binder; and $K_{p}$ and $\mu_{p}$ are the bulk and shear moduli of particles, respectively.

Fixing the axial strain $\bar{\varepsilon}$, the strain energy density $U$ of the RVE is a function of Legendre polynomial coefficients $\left[u_{n}^{I}\right]$ and $\left[v_{n}^{I}\right]$ given by Eqs. (1) to (4). By minimizing the strain energy density $U\left(u_{n}^{I}, v_{n}^{I}\right)$, fraction-balanced interface displacement jumps can be found.

After $u_{n}^{I}$ and $v_{n}^{I}$ are determined, the tensile stress $\bar{\sigma}$ can be obtained from Eq. (4). The volume dilatation can be calculated from

$$
\Delta=\frac{\bar{\sigma}}{3 K_{\text {perf }}}+3 e_{0} \varepsilon_{0}
$$

where $K_{\text {perf }}$ is the bulk modulus for the same composite with perfect interfaces,

$$
K_{\text {perf }}=\left(\frac{1}{K_{b}}+f e_{0}\left(\frac{1}{K_{p}}-\frac{1}{K_{b}}\right)\right)^{-1}
$$




\section{DETERMINING INTERFACE PROPERTIES OF REDESIGNED SOLID ROCKET MOTOR PROPELLANTS}

The ammonium perchlorate particles have a bimodal size distribution in the RSRM propellants. The volume fraction of coarse particles (size $200 \mu \mathrm{m}$ ) is $56 \%$, and that of fine particles (size $20 \mu \mathrm{m}$ ) is $14 \%$. The mechanical behavior of solid propellant depends on its microstructural properties. Effects of chemical properties, such as that of bonding agent, are reflected in the current model through the interface bonding parameters. Compared with the binder, particles are very hard and can be treated as rigid. During slow loading, the binder can be treated as linear elastic, with Young's modulus $E_{b}=2.4 \mathrm{MPa}$ and Poisson's ratio $\nu_{b}=0.4995$.

Figure 1 gives a set of experimental data of axial strain $\bar{\varepsilon}_{i}^{\text {test }}$, tensile stress $\bar{\sigma}_{i}^{\text {test }}$, and volume dilatation $\Delta_{i}^{\text {test }}$, where $i$ runs from 1 to $N_{\text {test }}\left(N_{\text {test }}\right.$ is the total number of test data). The error between the model prediction (based on the four interface properties, $\delta_{\text {open }}, \delta_{\text {slide }}, \gamma_{\text {int }}$, and $\lambda_{e}$ ) and experimental data is defined as

$$
\begin{aligned}
& \operatorname{Err}\left(\delta_{\text {open }}, \delta_{\text {slide }}, \gamma_{\text {int }}, \lambda_{e}\right) \\
&=\frac{1}{\left\langle\bar{\sigma}^{\text {test }}\right\rangle} \sqrt{\frac{1}{N_{\text {test }}} \sum_{i=1}^{N_{\text {test }}}\left[\bar{\sigma}_{i}^{\text {test }}-\bar{\sigma}^{\text {predict }}\left(\bar{\varepsilon}_{i}^{\text {test }}\right)\right]^{2}} \\
&+\frac{1}{\left\langle\Delta^{\text {test }}\right\rangle} \sqrt{\frac{1}{N_{\text {test }}} \sum_{i=1}^{N_{\text {test }}}\left[\Delta_{i}^{\text {test }}-\Delta^{\text {predict }}\left(\bar{\varepsilon}_{i}^{\text {test }}\right)\right]^{2}}
\end{aligned}
$$

where $\bar{\sigma}^{\text {predict }}\left(\bar{\varepsilon}_{i}^{\text {test }}\right)$ and $\Delta^{\text {predict }}\left(\bar{\varepsilon}_{i}^{\text {test }}\right)$ are the model predictions of the tensile stress and volume dilatation at axial strain $\bar{\varepsilon}_{i}^{\text {test }}$, respectively; $\left\langle\bar{\sigma}^{\text {test }}\right\rangle$ $=1 / N_{\text {test }} \sum_{i} \bar{\sigma}_{i}^{\text {test }}$, and $\left\langle\Delta^{\text {test }}\right\rangle=1 / N_{\text {test }} \sum_{i} \Delta_{i}^{\text {test }}$.

Interface properties are obtained by minimizing the error function $\operatorname{Err}\left(\delta_{\text {open }}\right.$, $\left.\delta_{\text {slide }}, \gamma_{\text {int }}, \lambda_{e}\right)$ which gives the critical interface opening $\delta_{\text {open }}=66 \mu \mathrm{m}$, the critical interface sliding $\delta_{\text {slide }}=0.79 \mathrm{~mm}$, the total cohesive energy $\gamma_{\text {int }}=33 \mathrm{~J} / \mathrm{m}^{2}$, and the linear coefficient $\lambda_{e}=2.3 \%$.

Figure 2 shows the stress-strain and the dilatation-strain curves with both model prediction and experimental data. The good matches between the predicted curves and the experimental data demonstrate that the interface cohesive law approximated using a combined measure $\lambda$ and a bilinear form of $\phi(\lambda)$ is appropriate for solid rocket propellants. 


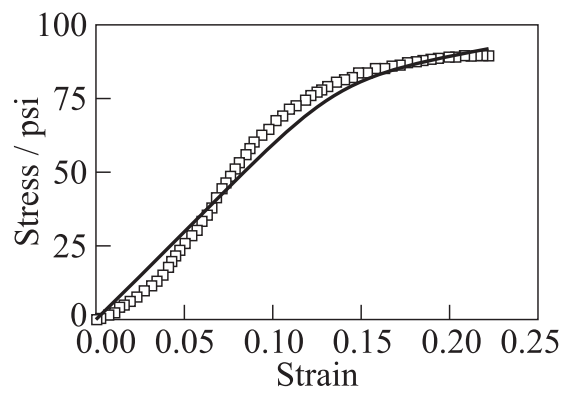

(a)

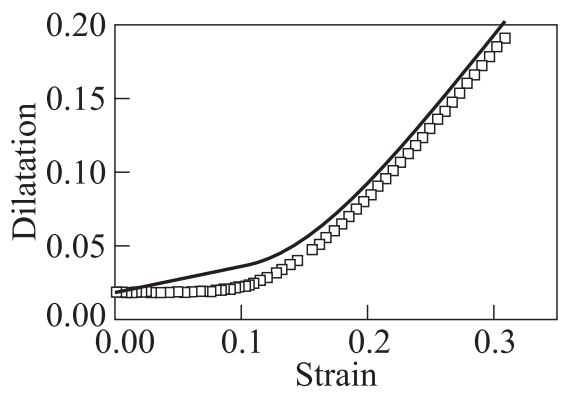

(b)

Figure 2 Calibration for interface parameters: $(a)$ relation between the engineering stress and strain; and $(b)$ volume dilatation-strain relation (signs - experimental data and curves - model prediction). The calibration of the interface parameters are: $\delta_{\text {open }}=66 \mu \mathrm{m}, \delta_{\text {slide }}=0.79 \mathrm{~mm}, \gamma_{\text {int }}=33 \mathrm{~J} / \mathrm{m}^{2}$, and $\lambda_{e}=2.3 \%$

\section{CONCLUDING REMARKS}

Using the micromechanics model that link the microscopic interface debonding with the macroscopic constitutive relations, the cohesive law for particle/binder interfaces in a reusable solid rocket motor propellant is determined based on the experiment data for the macroscopic stress-strain and dilation-strain relationships. The critical interface opening and sliding displacements are $\delta_{\text {open }}$ $=66 \mu \mathrm{m}$ and $\delta_{\text {slide }}=0.79 \mathrm{~mm}$, respectively. The interface cohesive energy is $\gamma_{\mathrm{int}}=33 \mathrm{~J} / \mathrm{m}^{2}$, and the linear coefficient $\lambda_{e}=2.3 \%$.

\section{ACKNOWLEDGMENTS}

This research was supported by the ASCI Center for Simulation of Advanced Rockets at the University of Illinois supported by U.S. Department of Energy through the University of California under subcontract No. B523819.

\section{REFERENCES}

1. Tan, H., Y. Huang, C. Liu, and P. H. Geubelle. 2005. The Mori-Tanaka method for composite materials with nonlinear interface debonding. Int. J. Plasticity 21:1890918.

2. Ho, S.Y., and C.W. Fong. 1987. Correlation between fracture properties and dynamic mechanical relaxations in composite propellants. Polymer 28:739-44. 
3. Sciammarella, C. A., and F. M. Sciammarella. 1998. Investigation of damage in solid propellants. 5th Conference (International) in Composites Engineering Proceedings. 779-80.

4. Ide, K. M., S.-Y. Ho, and D. R. G. Williams. 1999. Fracture behaviour of accelerated aged solid rocket propellants. J. Mater. Sci. 34:4209-18.

5. Tan, H., C. Liu, Y. Huang, and P. H. Geubelle. 2005. The cohesive law for the particle/matrix interfaces in high explosives. J. Mech. Phys. Solids 53:1892-917.

6. Canga, M.E., E. B. Becker, and S. Ozupek. 2001. Constitutive modeling of viscoelastic materials with damage - computational aspects. Comput. Methods Appl. Mech. Engrg. 190:2207-26.

7. Tvergaard, V., and J.W. Hutchinson. 1992. The relation between crack growth resistance and fracture process parameters in elastic-plastic solids. J. Mech. Phys. Solids 40:1377-97.

8. Tan, H., Y. Huang, C. Liu, G. Ravichandran, H. M. Inglis, and P. H. Geubelle. 2007. The uniaxial tension of particle-reinforced composite materials with nonlinear interface debonding. Int. J. Solids Struct. 44:1809-22.

9. Tan, H., Y. Huang, C. Liu, G. Ravichandran, and G.H. Paulino. 2007. Uniaxial tension of composites with nonlinear interface debonding: The Mori-Tanaka method. Int. J. Fract. 146:139-48. 